\title{
Cost-effective long-read assembly of a hybrid Formica aquilonia $\times$ Formica polyctena wood ant genome from a single haploid individual
}

\author{
Pierre Nouhaud ${ }^{1,2, *}$, Jack Beresford ${ }^{1,2} \&$ Jonna Kulmuni ${ }^{1,2}$ \\ ${ }^{1}$ Organismal \& Evolutionary Biology Research Programme, University of Helsinki, Helsinki, Finland \\ ${ }^{2}$ Tvärminne Zoological Station, University of Helsinki, Hanko, Finland \\ * Corresponding author: Pierre Nouhaud, Organismal \& Evolutionary Biology Research Programme, \\ University of Helsinki, Helsinki, Finland, pierr3.nouhaud@gmail.com
}

ORCiD IDs: PN: 0000-0003-4923-0993, JB: 0000-0002-2000-6085, JK: 0000-0002-8852-0793

\begin{abstract}
Formica red wood ants are a keystone species of boreal forest ecosystems and an emerging model system in the study of speciation and hybridization. Here we performed a standard DNA extraction from a single, field-collected Formica aquilonia $\times$ Formica polyctena haploid male and assembled its genome using $\sim 60 \times$ of PacBio long reads. After polishing and contaminant removal, the final assembly was 272 $\mathrm{Mb}(4,687$ contigs, $\mathrm{N} 50=1.16 \mathrm{Mb})$. Our reference genome contains $98.5 \%$ of the core Hymenoptera BUSCOs and was scaffolded using the pseudo-chromosomal assembly of a related species, F. selysi (28 scaffolds, N50 = 8.49 Mb). Around one third of the genome consists of repeats, and 17,426 gene models were annotated using both protein and RNAseq data (97.4\% BUSCO completeness). This resource is of comparable quality to the few other single individual insect genomes assembled to date and paves the way to genomic studies of admixture in natural populations and comparative genomic approaches in Formica wood ants.
\end{abstract}

Keywords Hymenoptera, genome assembly, genome annotation, PacBio sequencing, haplodiploidy, wood ant 


\section{INTRODUCTION}

Despite their small size, red wood ants (subgenus Formica sensu stricto, or F. rufa group, hereafter wood ants) are heavyweights of boreal ecosystems. These social insects build massive interconnected nest mounds forming supercolonies of several million individuals, covering up to $2 \mathrm{~km}^{2}$ (Stockan, Robinson, Trager, Yao, \& Seifert, 2016). Wood ants are considered keystone species which play a role in nutrient cycling (Frouz, Jílková, \& Sorvari, 2016), predator-prey dynamics or plant growth (Robinson, Stockan, \& lason, 2016), to name a few.

Wood ant genomics have so far mostly focused on supercoloniality, which is an extreme form of sociality. While the canonical ant colony is headed by a single queen (monogyny) and occupies a unique nest, in wood ant supercolonies a single nest may contain dozens to hundreds of unrelated egg-laying queens (polygyny, Pamilo, 1993). This social polymorphism is governed by a supergene maintained across species which diverged 40 Mya (Brelsford et al., 2020; Purcell, Brelsford, Wurm, Perrin, \& Chapuisat, 2014).

Wood ants have undergone recent radiation (Goropashnaya, Fedorov, Seifert, \& Pamilo, 2012) and represent a promising system for the study of speciation and hybridization. This process is ubiquitous across living organisms and haplodiploids (organisms for which one sex is haploid and the other, diploid) such as wood ants can answer some key questions in admixture research which are difficult to study in standard diploid organisms (Nouhaud, Blanckaert, Bank, \& Kulmuni, 2020). The bestcharacterised case is the occurrence of natural hybrids between $F$. aquilonia and $F$. polyctena in Southern Finland. Two hybrid lineages coexist in a single population (Kulmuni, Seifert, \& Pamilo, 2010), where introgression between lineages is sex-specific but could be modulated by external factors (Kulmuni et al., 2020).

Currently, no high-quality reference genome is available for any species of the Formica s. str. subgenus. Kulmuni et al. (2020) assembled a draft genome using poolseq data from a hybrid $F$. aquilonia $\times F$. polyctena population, but the assembly is highly fragmented ( $>300 \mathrm{k}$ contigs, $\mathrm{N} 50<2 \mathrm{kbp}$ ). At a broader phylogenetic scale, among palaearctic Formica species, two genomes are available for the Coptoformica and Serviformica subgenera, respectively F. exsecta (Dhaygude, Nair, Johansson, Wurm, \& Sundström, 2019) and F. selysi (Brelsford et al., 2020). However, divergence is around 10\% between each subgenus pair (Goropashnaya et al., 2012), which precludes using either of these genomes as references when studying members of the Formica s. str. clade.

While PacBio DNA input requirements have for a long time hindered the individual sequencing of small organisms, a modified SMRTbell library construction protocol was recently used to build a reference genome from a single Anopheles mosquito (Kingan, Heaton, et al., 2019). Few other recent examples demonstrate that high-quality arthropod genomes can now be built from a single individual (fruit fly: Adams et al., 2020; lanternfly: Kingan, Urban, et al., 2019; braconid wasp: Ye et al., 2020). Here, we assemble the genome of a single haploid, hybrid F. aquilonia $\times$ F. polyctena male using PacBio sequencing. As sexuals from these species are relatively big $(\sim 20 \mathrm{mg})$, we could apply a cost-effective, standard extraction protocol to obtain high-molecular weight DNA from a single individual. The contigs were anchored against the $F$. selysi chromosomal assembly after contamination removal, and the genome was annotated using both RNAseq and protein data. Overall, the quality of the assembly and 
its annotation are on par with other single individual insect genomes published to date as well as other sequenced ant genomes.

\section{MATERIALS \& METHODS}

\section{Sampling}

All individuals used in the present study were sampled from the Långholmen population in Southern Finland $\left(59^{\circ} 50^{\prime} 59.9^{\prime \prime} \mathrm{N}, 23^{\circ} 15^{\prime} 03.3^{\prime \prime} \mathrm{E}\right)$ in Spring 2018 . This population has been characterized as a hybrid between $F$. aquilonia and $F$. polyctena using both genetic markers and morphological data (Kulmuni et al., 2010; Seifert, Kulmuni, \& Pamilo, 2010). The Långholmen population is a supercolony consisting of two genetic lineages of hybrid origin (R and W; (Kulmuni \& Pamilo, 2014; Kulmuni et al., 2010 ), which show moderate genetic differentiation ( $F_{S T} \approx 0.105$, Kulmuni et al., 2020).

For long-read sequencing, a single haploid male was collected from the FAu2014a nest (W lineage) in Spring 2018. Two males and two unmated gynes (queens) from the same nest and lineage were also sampled at the same time for polishing purposes (short-read sequencing, see below). All samples were collected in individual sterile tubes and flash-frozen on the field. For RNA sequencing, sexual larvae were collected from multiple R and W nests in the same population in May 2014, measured and put in individual tubes before flash-freezing in the laboratory within 24 hours of collection (Beresford et al., unpublished). All samples were stored at $-80^{\circ} \mathrm{C}$ without any buffer.

\section{Long-read sequencing}

For both PacBio and Illumina DNA sequencing, all steps were carried by Novogene (Hong Kong) as part of the Global Ant Genomics Alliance (GAGA, Boomsma et al., 2017). DNA from a single haploid male was extracted using a Sodium Dodecyl Sulfate (SDS) protocol, and a SMRTbell library was prepared using the SMRT bell Template Prep Kit 1.0-SPv3 (Pacbio, 100-991-900). DNA quantification was performed using a Qubit fluorometer (Thermo Fisher) and purity was assessed with an agarose gel electrophoresis. The extraction from a single male yielded $9.89 \mu \mathrm{g}$ of DNA, at a concentration of 86 $\mathrm{ng} \cdot \mathrm{Il}^{-1}\left(\mathrm{~A}_{260 / 280}=1.76, \mathrm{~A}_{260 / 230}=1.20\right)$. DNA fragmentation was assessed through an Advanced Analytical Fragment Analyzer (AATI, mean size: 18317 bp) prior to size selection (BluePippin, Sage Sciences, cutoff: $10 \mathrm{~kb}$ ). The sample was loaded onto four SMRT cells with the Sequel Sequencing Kit 2.0 following PacBio recommendations and sequenced on a PacBio Sequel platform.

\section{Short-read DNA sequencing}

Since accuracy of long-read data is lower than short-read data (e.g., Koren et al., 2012; but see Wenger et al., 2019), Illumina data was generated to correct spurious base calls. For the four samples used for these polishing purposes, DNA was extracted from whole bodies with a SDS protocol and libraries were constructed using NEBNext DNA Library Prep Kits (New England Biolabs). Whole-genome sequencing was performed on Illumina Novaseq 6000 (paired-end mode, 150 bp), after which raw Illumina reads and adapter sequences were trimmed using Trimmomatic (v0.38; parameters LEADING:20 TRAILING:20 MINLEN:50; Bolger, Lohse, \& Usadel, 2014). 


\section{Whole-genome assembly}

We assessed the performance of two long-read assemblers, Canu (v1.8, Koren et al., 2017) and wtdbg2 (v2.5, Ruan \& Li, 2020). We assumed a haploid genome size of $323 \mathrm{Mb}$, which is the mean size estimated from five species of the Formicinae subfamily by flow cytometry (Tsutsui, Suarez, Spagna, \& Johnston, 2008). Canu was run with default parameters, except that the maximum allowed difference threshold was adapted to Sequel data (correctedErrorRate=0.085), following Canu's FAQ. For wtdbg2, a first run was performed using settings optimised for Sequel data and genome sizes below $1 \mathrm{~Gb}$ (preset 2: -p 0 -k 15 -AS 2 -s 0.05) but selecting all subread lengths (-L 0$)$. Based on the subread distribution, a second run was performed with the same preset, but selecting only subreads above 10 $\mathrm{kb}$ (-L 10000). For each assembly we assessed completeness using BUSCO (v4.0.5, Seppey, Manni, \& Zdobnov, 2019) with the Hymenoptera ODB gene set v10.

The canu assembly contained $338 \mathrm{Mb}$ in 3,633 contigs (assuming a haploid genome size of $323 \mathrm{Mb}$, NG50 = $283 \mathrm{~kb}$ ), the wtdbg2 assembly using all subreads totaled $349 \mathrm{Mb}$ in 11,615 contigs (NG50 = $71 \mathrm{~kb})$ and running wtdbg2 only with subreads greater than $10 \mathrm{~kb}(\sim 44 \times)$ yielded a $280 \mathrm{Mb}$ assembly with 5,098 contigs (NG50 = $689 \mathrm{~kb}$ ). Because of a large fraction of missing BUSCOs, the wtdbg2-all assembly was discarded (supplementary table 1). The completeness of both canu and wtdbg2-10k assemblies were comparable ( $97.5 \%$ vs. $97.1 \%$, respectively) despite stark differences in total sizes (338 Mb vs. $280 \mathrm{Mb}$, respectively). However, the BUSCO duplication rate was much higher for the canu assembly ( $5.7 \%$ vs. $0.5 \%$ ). This suggests that the canu assembly may contain duplicated regions, which could in turn inflate its size. Interestingly, while the average genome size for the Formicinae subfamily was estimated at $323 \mathrm{Mb}$ by flow cytometry (Tsutsui et al., 2008), recent genome projects within the Formica genus documented genome sizes much closer to our $280 \mathrm{Mb}$ estimate, with 278 Mb for F. exsecta (Dhaygude et al., 2019) and $290 \mathrm{Mb}$ for F. selysi (Brelsford et al., 2020). Based on this observation, plus the assembly statistics and BUSCO score (supplementary table 1), we concluded that the wtdbg2-10k assembly was the best model. The next steps were only performed on this assembly.

\section{Assembly polishing}

To avoid incorporating sequencing errors in our final assembly (Watson \& Warr, 2019), we polished our contigs using Racon (v1.4.10, Vaser, Sović, Nagarajan, \& Šikić, 2017). We ran four polishing iterations with the PacBio data, followed by two iterations with the Illumina resequencing data (all four individuals pooled), always keeping unpolished sequences in the output (parameter $-\mathrm{u}$ ). For each iteration, alignment was performed using minimap2 (v2.17, Li, 2016, using parameters -x map-pb for PacBio and -ax sr for Illumina data, respectively).

\section{Contaminant removal \& mitochondrial genome identification}

The assembly was assessed for contaminants with BlobTools (v1.1.1, Laetsch \& Blaxter, 2017). Coverage files were obtained using minimap2 for both Canu-corrected PacBio subreads and the four resequenced individuals. Taxonomic partitioning of contigs was carried through BLAST against the 
NCBI non-redundant database. The contig containing the mitochondrial genome was identified based on BlobTools results (lower GC proportion compared to the rest of the genome and high sequencing depth, supplementary fig. 1) and was further validated by BLAST of the F. selysi mtDNA sequence (Brelsford et al., 2020) against the whole assembly. Formica ants carry Wolbachia endosymbionts (Viljakainen, Reuter, \& Pamilo, 2008) and horizontal gene transfer (HGT) has been previously characterized in F. exsecta (Dhaygude et al., 2019). To avoid classifying ant contigs impacted by HGT as contigs of endosymbiont origin, we blasted the closest Wolbachia genome (NCBI accession PRJNA436771) against our assembly and manually inspected these results in conjunction with coverage profiles and the physical location of Hymenoptera BUSCO hits (v4.0.5, Seppey et al., 2019).

\section{Anchoring of contigs to pseudo-chromosomes}

Our polished, ant nuclear contigs were coalesced into pseudo-chromosomes with RaGOO (v1.1, Alonge et al., 2019), using the F. selysi reference genome (Brelsford et al., 2020) as a guide. To evaluate RaGOO's performance, we also aligned contigs against $F$. selysi pseudo-chromosomes using the nucmer aligner from MUMmer (v4.0.0beta2, Marçais et al., 2018). Delta files from nucmer were processed using the DotPrep.py script (https://dnanexus.github.io/dot/DotPrep.py, last accessed 20.10.2020) and alignments were visualised using Dot (https://dot.sandbox.bio/, last accessed 20.10.2020). A large portion (6 Mb) of Scaffold 10 in F. selysi mostly contained highly repetitive alignments (see also Figure 1 from Brelsford et al., 2020). This region was removed from the $F$. selysi assembly before a second RaGOO run was performed. The gap size was set to $100(100 \times \mathrm{N})$. All remaining, unanchored contigs were scaffolded as a single Scaffold 0 . Of note, both parental species $F$. aquilonia and $F$. polyctena have 26 chromosomes $(n=26$, Rosengren \& Rosengren, 1980), while $F$. selysi has $27(n=27)$. Unfortunately, the breakpoint could not be identified on the sole basis of our long-read data. Our assembly contains 27 pseudo-chromosomes instead of the 26 , which is the correct karyotype for both parental species.

\section{Annotation of repeat sequences}

Transposable elements (TEs) were annotated using the Dfam TE Tools Container (v1.1, https:/github.com/Dfam-consortium/TETools, last accessed 20.10.2020). A de novo consensus library was built with Repeatmodeler 2 (Flynn et al., 2020) and used to mask TE sequences in our assembly using Repeatmasker (Smit, Hubley, \& Green, 2013).

\section{RNA sequencing}

For annotation purposes, RNAseq data was generated for nine individuals originating from six nests in the Långholmen population (R: nest FA4, 3 individuals and W: nest FA15, 1 ind.; FA17, 2 inds.; FA25, 1 ind.; FA35, 1 ind.; FAu2014a, 1 ind.). These individuals were at different larval stages and total RNA was extracted from whole bodies using an ALLPrep DNA/RNA Mini Kit (Qiagen) following manufacturer's instructions. Individual RNA qualities were assessed using a Bioanalyzer (Agilent 2100). Libraries were constructed using NEBNext Ultra RNA Library Prep Kits and samples were sequenced on an Illumina NextSeq platform (paired-end mode, $150 \mathrm{bp}$ ) at the Biomedicum Functional Genomics 
Unit (FuGU, University of Helsinki). Raw reads were trimmed using Trimmomatic (v0.38, parameters LEADING:20 TRAILING:20 SLIDINGWINDOW:5:20 MINLEN:50 Bolger et al., 2014) and unpaired reads were discarded. Approximately 5.60 million $150 \mathrm{bp}$ paired reads were randomly sampled per individual and combined into two (Forward and Reverse) FASTQ files, totaling 50 million paired reads over all individuals.

\section{Genome annotation}

We annotated the genome with the Braker2 pipeline (Brůna, Hoff, Lomsadze, Stanke, \& Borodovsky, 2020; v2.1.5, Hoff, Lomsadze, Borodovsky, \& Stanke, 2019). Both RNAseq- and protein-derived hints were used to train GeneMark-ETP, which predictions were in turn used to train Augustus and obtain the final gene set. All protein data available for Arthropoda were downloaded from OrthoDB (v10, Kriventseva et al. 2019, https://v100.orthodb.org/download/odb10_arthropoda_fasta.tar.gz, last accessed 22.07.2020) and aligned using ProtHint. This dataset contains 2.6 million sequences and encompasses 170 species, including 40 of the same order (Hymenoptera) and 17 of the same family (Formicidae). RNAseq data produced above was aligned against the hard-masked genome using STAR (v2.7.2, Dobin et al., 2013), and secondary alignments were removed with SAMtools (v1.10, Li et al., 2009). After the Braker2 run, protein sequences of all gene models not supported by at least one hint were blasted against the Uniprot database (UniProt Consortium, 2019) and all models without any hit on Aculeata (wasps, bees and ants) were discarded from the final gene set. Finally, functional annotation was carried with EnTAP (v0.10.3, Hart et al., 2020) using the EggNOG (Huerta-Cepas et al., 2016), Uniprot and RefSeq databases.

\section{RESULTS AND DISCUSSION}

\section{Genome sequencing \& assembly}

We generated $2,547,044$ subreads on the PacBio Sequel, suming to $21.8 \mathrm{~Gb}$ of data $(\sim 68 \times)$. Half of the subreads were longer than $11.5 \mathrm{~kb}$ (NR50), with a mean length of $8.55 \mathrm{~kb}$.

Running wtdbg2 only with subreads greater than $10 \mathrm{~kb}(\sim 44 \times)$ yielded a $280 \mathrm{Mb}$ assembly with 5,098 contigs (assuming a haploid genome size of $323 \mathrm{Mb}, \mathrm{NG50}=689 \mathrm{~kb}$, supplementary table 1). Interestingly, while the average genome size for the Formicinae subfamily was estimated at $323 \mathrm{Mb}$ by flow cytometry (Tsutsui et al., 2008), recent genome projects within the Formica genus documented genome sizes much closer to our $280 \mathrm{Mb}$ estimate, with $278 \mathrm{Mb}$ for $F$. exsecta (Dhaygude et al., 2019) and $290 \mathrm{Mb}$ for $F$. selysi (Brelsford et al., 2020). Based on this observation (similar assembly sizes for different Formica species) and BUSCO metrics, we concluded that our assembly had a sufficiently high level of completeness.

After polishing using both long (four iterations) and short reads (two iterations), the BUSCO score reached $98.5 \%$ for complete single-copy orthologs (table 1 ) while the total size of the assembly reduced to $276 \mathrm{Mb}$. 
Table 1. Assembly \& annotation metrics. *: scaffold statistics computed after excluding both the mitochondrial genome and Scaffold 0 , which contains all unanchored contigs (59 Mb, "total unanchored length").

Genome assembly

BUSCO v4.0.5 genome score

Number of contigs

Contig N50 (bp)

Shortest contig (bp)

Longest contig (bp)

Average contig length (bp)

Total contig length (bp)

Number of scaffolds

Scaffold N50* (bp)

Shortest scaffold (bp)

Longest scaffold* (bp)

Average scaffold length* (bp)

Total scaffold length (bp)

Total unanchored length (bp, fraction)

GC content

$\mathrm{N}$ fraction

\section{Genome annotation}

BUSCO v4.0.5 protein score

Total number of gene models

Mean gene length (bp)

Average number of exons per gene

Number of models with RNAseq support (fraction)

Number of isoforms

Average number of isoforms per gene

Cumulative gene length ( $b p$, fraction)

Cumulative exon length (bp, fraction)

\section{Repeat annotation}

Fraction of genome masked

$32.01 \%$

Interspersed repeats, total fraction

$28.44 \%$

Retroelements (class I)

$6.39 \%$

LINEs

$1.47 \%$

Gypsy/DIRS1

$2.72 \%$

DNA transposons (class II)

$3.56 \%$

Unclassified

$18.50 \%$

Simple repeats
C:98.5\%[S:97.9\%,D:0.6\%], F:0.4\%,M:1.1\%,n:5991$$
163,114
$$

117

$4,650,116$

58,036

$272,015,305$

$8,490,488$

$3,646,393$

$7,887,222$

$272,497,664$

$36.3 \%$

$0.17 \%$

C:97.4\%[S:96.8\%,D:0.6\%], F:1.4\%,M:1.2\%,n:5991

17,426

5,524

5.80

19,226

1.10

$78,835,002(29.0 \%)$

$27,442,032(10.1 \%)$ 
Almost $92 \%(4,688)$ of the 5,098 contigs were assigned to Arthropoda, while 82 contigs were assigned to Proteobacteria (supplementary fig. 1). Formica ants harbor Wolbachia endosymbionts (Viljakainen et al., 2008), and HGT between Wolbachia and the ant nuclear genome has been characterized (Dhaygude et al., 2019). Through manual curation we assigned 76 contigs to Wolbachia (total size = $1,786,664 \mathrm{bp}, \mathrm{N} 50=33.4 \mathrm{~kb}$ ) and six contigs of the nuclear ant genome as putative HGTs. Overall, the contamination removal step decreased the nuclear ant genome size to 272,015,305 bp.

Finally, we anchored $78.2 \%$ (213 Mb, table 1) of our assembly to the 27 pseudo-chromosomes of the F. selysi genome, a fraction similar to that of the original F. selysi study $(78.3 \%$ of the assembly assigned to pseudo-chromosomes, see Table S3 in Brelsford et al., 2020).

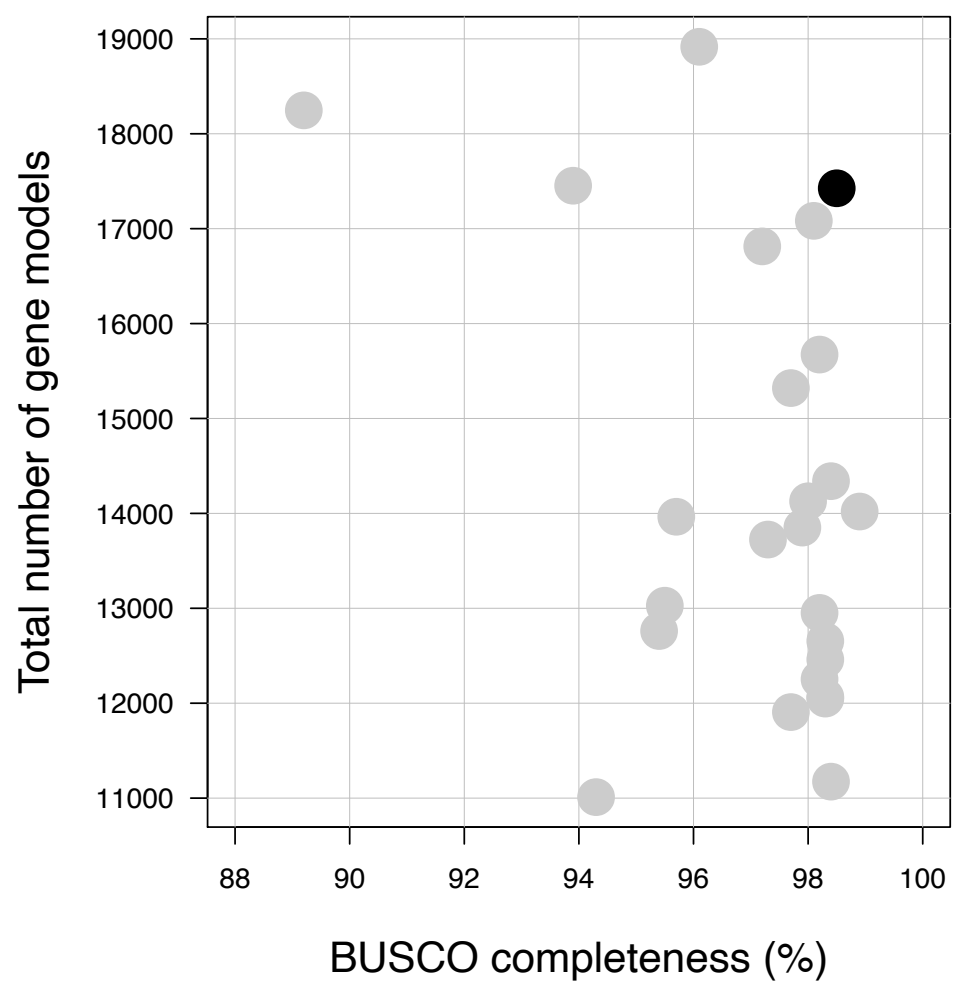

Fig. 1. Total number of gene models as a function of BUSCO genome completeness metrics in ant genomes for which annotations are available on $\mathrm{NCBI}(n=24$, light gray) and the assembly of this study (black). Detailed statistics are shown in supplementary table 3 .

\section{Genome annotation}

Overall, $32 \%$ of the sequence was masked with Repeatmasker, most of the repeats being unclassified (18.5\%), 6.39\% being retroelements and $3.56 \%$ being DNA transposons (table 1 ). The vast majority of repeats were located on unanchored contigs (supplementary fig. 2).

The initial gene set contained 30,068 gene models, which is far superior to what has been documented in ants ( 17,000 gene models, Gadau et al., 2012). Among these models, 14,287 (47.5\%) were not supported by any protein or RNAseq hint. Moreover, the size of these hint-less models was much shorter than hint-supported models. As we suspected an overprediction problem (which was also observed for alternative Braker2 runs, supplementary table 2), we only kept hint-less models if their protein sequences had a blast hit against Aculeata in Uniprot, which reduced the total set from 30,068 
to 17,426 gene models (15,781 with hints plus 1,645 recovered after blast). Overall, 19,226 mRNAs were identified, among which 15,664 (81.5\%) were functionally annotated with EnTAP. From these, $63.4 \%$ of the proteins had their best hit within ant species or Drosophila melanogaster (supplementary fig. 3). The completeness of this final gene set assessed with BUSCO was good (protein mode: $97.4 \%$, table 1) and our assembly showed a level of completeness comparable to other ant genomes annotated so far (fig. 1 , supplementary table 3).

\section{Conclusions}

Here we report a pseudo-chromosome-level genome assembly for a single hybrid $F$. aquilonia $\times F$. polyctena haploid male using a simple and cost-effective extraction protocol. The final assembly sums to $272 \mathrm{Mb}$, of which $78.2 \%$ are anchored onto 27 scaffolds, and recovers $98.5 \%$ of Hymenopteraspecific single-copy orthologs. Our annotation contains 17,426 protein-coding genes, with a BUSCO completeness of $97.4 \%$.

Previously published single insect genomes have used either Nanopore or PacBio sequencing, sometimes coupled with whole genome amplification or DNA extraction tailored to small starting material. We used standard extraction protocol from haploid tissue with PacBio sequencing and produced haploid reference genome reaching similar BUSCO and N50 statistics as previous single insect genomes (supplementary table 4).

This work provides a crucial resource to study speciation and contemporary hybridization in the Formica rufa group, as well as the evolution of extreme sociality. It will also enable new approaches on the genomics of hybridization in this fascinating system. Finally, it also demonstrates that high-quality arthropod genomes can be assembled from single individuals using standard, cost-effective protocols.

\section{ACKNOWLEDGMENTS}

We thank Alan Brelsford for sharing the F. selysi assembly and we acknowledge Daniel Blande, Dominik Laetsch, Alex Mackintosh and Yannick Wurm for feedback. We are grateful to CSC - IT Center for Science, Finland, for computational resources. This work was performed under the umbrella of the Global Ant Genomic Alliance and was supported by an HiLIFE fellowship and an Academy of Finland grant no. 309580 to JK.

\section{AUTHOR CONTRIBUTIONS}

PN \& JK designed the project. PN, JB \& JK performed sampling. JB \& JK generated the RNAseq data. PN carried all analyses and drafted the manuscript, which all authors revised for important intellectual content.

\section{DATA ACCESSIBILITY}

The genome assembly has been deposited at the European Nucleotide Archive under the study PRJEB41943. The gene annotation, associated protein sequences and RNAseq data used for annotation purposes are available on Figshare (doi: 10.6084/m9.figshare.c.5332442.v1 \& 10.6084/m9.figshare.c.5277767). 


\section{REFERENCES}

Adams, M., McBroome, J., Maurer, N., Pepper-Tunick, E., Saremi, N. F., Green, R. E., ... Corbett-Detig, R. B. (2020). One fly-one genome: chromosome-scale genome assembly of a single outbred Drosophila melanogaster. Nucleic Acids Research, 48(13), e75.

Alonge, M., Soyk, S., Ramakrishnan, S., Wang, X., Goodwin, S., Sedlazeck, F. J., ... Schatz, M. C. (2019).

RaGOO: fast and accurate reference-guided scaffolding of draft genomes. Genome Biology, 20(1), 224.

Bolger, A. M., Lohse, M., \& Usadel, B. (2014). Trimmomatic: a flexible trimmer for Illumina sequence data. Bioinformatics , 30(15), 2114-2120.

Boomsma, J. J., Brady, S. G., Dunn, R. R., Gadau, J., Heinze, J., Keller, L., ... Zhang, G. (2017). The Global Ant Genomics Alliance (GAGA). Myrmecological News / Osterreichische Gesellschaft Fur Entomofaunistik, 25, 6166.

Brelsford, A., Purcell, J., Avril, A., Tran Van, P., Zhang, J., Brütsch, T., ... Chapuisat, M. (2020). An Ancient and Eroded Social Supergene Is Widespread across Formica Ants. Current Biology: CB, 30(2), 304-311.e4.

Brůna, T., Hoff, K. J., Lomsadze, A., Stanke, M., \& Borodovsky, M. (2020). BRAKER2: Automatic Eukaryotic Genome Annotation with GeneMark-EP+ and AUGUSTUS Supported by a Protein Database (p. 2020.08.10.245134). doi: 10.1101/2020.08.10.245134

Dhaygude, K., Nair, A., Johansson, H., Wurm, Y., \& Sundström, L. (2019). The first draft genomes of the ant Formica exsecta, and its Wolbachia endosymbiont reveal extensive gene transfer from endosymbiont to host. BMC Genomics, 20(1), 301.

Dobin, A., Davis, C. A., Schlesinger, F., Drenkow, J., Zaleski, C., Jha, S., ... Gingeras, T. R. (2013). STAR: ultrafast universal RNA-seq aligner. Bioinformatics , 29(1), 15-21.

Flynn, J. M., Hubley, R., Goubert, C., Rosen, J., Clark, A. G., Feschotte, C., \& Smit, A. F. (2020). RepeatModeler2 for automated genomic discovery of transposable element families. Proceedings of the National Academy of Sciences of the United States of America, 117(17), 9451-9457.

Frouz, J., Jílková, V., \& Sorvari, J. (2016). Contribution of wood ants to nutrient cycling and ecosystem function. In Wood Ant Ecology and Conservation (pp. 207-220). Cambridge University Press.

Gadau, J., Helmkampf, M., Nygaard, S., Roux, J., Simola, D. F., Smith, C. R., ... Smith, C. D. (2012). The genomic impact of 100 million years of social evolution in seven ant species. Trends in Genetics: TIG, 28(1), 14-21.

Goropashnaya, A. V., Fedorov, V. B., Seifert, B., \& Pamilo, P. (2012). Phylogenetic relationships of Palaearctic Formica species (Hymenoptera, Formicidae) based on mitochondrial cytochrome B sequences. PloS One, 7(7), e41697.

Hart, A. J., Ginzburg, S., Xu, M. (sam), Fisher, C. R., Rahmatpour, N., Mitton, J. B., ... Wegrzyn, J. L. (2020). EnTAP : Bringing faster and smarter functional annotation to non-model eukaryotic transcriptomes. Molecular Ecology Resources, 20(2), 591-604.

Hoff, K. J., Lomsadze, A., Borodovsky, M., \& Stanke, M. (2019). Whole-Genome Annotation with BRAKER. In M. Kollmar (Ed.), Gene Prediction: Methods and Protocols (pp. 65-95). New York, NY: Springer New York.

Huerta-Cepas, J., Szklarczyk, D., Forslund, K., Cook, H., Heller, D., Walter, M. C., .. Bork, P. (2016). eggNOG 4.5: a hierarchical orthology framework with improved functional annotations for eukaryotic, prokaryotic and viral sequences. Nucleic Acids Research, 44(D1), D286-D293.

Kingan, S. B., Heaton, H., Cudini, J., Lambert, C. C., Baybayan, P., Galvin, B. D., ... Lawniczak, M. K. N. (2019). A High-Quality De novo Genome Assembly from a Single Mosquito Using PacBio Sequencing. Genes, 10(1), 62.

Kingan, S. B., Urban, J., Lambert, C. C., Baybayan, P., Childers, A. K., Coates, B., ... Geib, S. M. (2019). A highquality genome assembly from a single, field-collected spotted lanternfly (Lycorma delicatula) using the PacBio Sequel II system. GigaScience, 8(10). doi: 10.1093/gigascience/giz122

Koren, S., Schatz, M. C., Walenz, B. P., Martin, J., Howard, J. T., Ganapathy, G., ... Adam M Phillippy. (2012). Hybrid error correction and de novo assembly of single-molecule sequencing reads. Nature Biotechnology, 30(7), 693-700.

Koren, S., Walenz, B. P., Berlin, K., Miller, J. R., Bergman, N. H., \& Phillippy, A. M. (2017). Canu: scalable and accurate long-read assembly via adaptive k-mer weighting and repeat separation. Genome Research, 27(5), 722-736.

Kulmuni, J., Nouhaud, P., Pluckrose, L., Satokangas, I., Dhaygude, K., \& Butlin, R. K. (2020). Instability of natural selection at candidate barrier loci underlying speciation in wood ants. Molecular Ecology, 29(20), 3988-3999.

Kulmuni, J., \& Pamilo, P. (2014). Introgression in hybrid ants is favored in females but selected against in males. Proceedings of the National Academy of Sciences of the United States of America, 111(35), 12805-12810. 
Kulmuni, J., Seifert, B., \& Pamilo, P. (2010). Segregation distortion causes large-scale differences between male and female genomes in hybrid ants. Proceedings of the National Academy of Sciences of the United States of America, 107(16), 7371-7376.

Laetsch, D. R., \& Blaxter, M. L. (2017). BlobTools: Interrogation of genome assemblies. F1000Research, 6(1287), 1287.

Li, H. (2016). Minimap and miniasm: fast mapping and de novo assembly for noisy long sequences. Bioinformatics , 32(14), 2103-2110.

Li, H., Handsaker, B., Wysoker, A., Fennell, T., Ruan, J., Homer, N., .. 1000 Genome Project Data Processing Subgroup. (2009). The Sequence Alignment/Map format and SAMtools. Bioinformatics , 25(16), 2078-2079.

Marçais, G., Delcher, A. L., Phillippy, A. M., Coston, R., Salzberg, S. L., \& Zimin, A. (2018). MUMmer4: A fast and versatile genome alignment system. PLoS Computational Biology, 14(1), e1005944.

Nouhaud, P., Blanckaert, A., Bank, C., \& Kulmuni, J. (2020). Understanding Admixture: Haplodiploidy to the Rescue. Trends in Ecology \& Evolution, 35(1), 34-42.

Pamilo, P. (1993). Polyandry and allele frequency differences between the sexes in the ant Formica aquilonia. Heredity, 70(5), 472-480.

Purcell, J., Brelsford, A., Wurm, Y., Perrin, N., \& Chapuisat, M. (2014). Convergent genetic architecture underlies social organization in ants. Current Biology: CB, 24(22), 2728-2732.

Robinson, E. J. H., Stockan, J. A., \& lason, G. R. (2016). Wood ants and their interaction with other organisms. In Wood Ant Ecology and Conservation (pp. 177-206). Cambridge University Press.

Rosengren, M., \& Rosengren, R. (1980). Chromosome numbers in the genus Formica with special reference to the taxonomical position of Formica uralensis Ruzsk. and Formica truncorum Fabr. Hereditas, 92, 321-325.

Ruan, J., \& Li, H. (2020). Fast and accurate long-read assembly with wtdbg2. Nature Methods, 17(2), 155-158.

Seifert, B., Kulmuni, J., \& Pamilo, P. (2010). Independent hybrid populations of Formica polyctena X rufa wood ants (Hymenoptera: Formicidae) abound under conditions of forest fragmentation. Evolutionary Ecology, 24(5), 1219-1237.

Seppey, M., Manni, M., \& Zdobnov, E. M. (2019). BUSCO: Assessing Genome Assembly and Annotation Completeness. In M. Kollmar (Ed.), Gene Prediction: Methods and Protocols (pp. 227-245). New York, NY: Springer New York.

Smit, A. F., Hubley, R., \& Green, P. (2013). Repeat-Masker Open-4.0. Retrieved October 20, 2020, from http://www.repeatmasker.org

Stockan, J. A., Robinson, E. J. H., Trager, J. C., Yao, I., \& Seifert, B. (2016). Introducing wood ants: evolution, phylogeny, identification and distribution. In Wood Ant Ecology and Conservation (pp. 1-36). Cambridge University Press.

Tsutsui, N. D., Suarez, A. V., Spagna, J. C., \& Johnston, J. S. (2008). The evolution of genome size in ants. BMC Evolutionary Biology, 8, 64.

UniProt Consortium. (2019). UniProt: a worldwide hub of protein knowledge. Nucleic Acids Research, 47(D1), D506-D515.

Vaser, R., Sović, I., Nagarajan, N., \& Šikić, M. (2017). Fast and accurate de novo genome assembly from long uncorrected reads. Genome Research, 27(5), 737-746.

Viljakainen, L., Reuter, M., \& Pamilo, P. (2008). Wolbachia transmission dynamics in Formica wood ants. BMC Evolutionary Biology, 8(1), 55.

Watson, M., \& Warr, A. (2019). Errors in long-read assemblies can critically affect protein prediction. Nature biotechnology, 37(2), 124-126.

Wenger, A. M., Peluso, P., Rowell, W. J., Chang, P.-C., Hall, R. J., Concepcion, G. T., ... Hunkapiller, M. W. (2019). Accurate circular consensus long-read sequencing improves variant detection and assembly of a human genome. Nature Biotechnology, 37(10), 1155-1162.

Ye, X., Yang, Y., Tian, Z., Xu, L., Yu, K., Xiao, S., .. Ye, G. (2020). A high-quality de novo genome assembly from a single parasitoid wasp (p. 2020.07.13.200725). doi: 10.1101/2020.07.13.200725 